\title{
Coherently controlled quantum emitters in cavities
}

\author{
A. Muller, ${ }^{a}$ E. B. Flagg, ${ }^{a}$ D. G. Deppe, ${ }^{b}$ G. J. Salamo, ${ }^{c}$ and C. K. Shih ${ }^{\mathrm{a}, *}$ \\ ${ }^{a}$ Department of Physics, The University of Texas at Austin; \\ ${ }^{\mathrm{b}}$ CREOL, University of Central Florida; \\ ${ }^{\mathrm{c}}$ Department of Physics, University of Arkansas
}

\begin{abstract}
Semiconductor nanostructures such as quantum dots (QDs) have offered unique opportunities to investigate quantum optical effects in solid-state systems. These include quantum interference, Rabi oscillations, as well as photon antibunching, and were previously observable only in isolated atoms or ions. In addition, QDs can be integrated into optical microcavities, making them attractive for applications in quantum information processing and high efficiency quantum light sources. Despite much progress towards these goals, one area that was little explored is coherent control of such solid-state quantum emitters in cavities. The main technical hurdle lies in overcoming the laser background scattering. By using a sample structure in which QDs are embedded in a planar Fabry-Perot cavity and by using an orthogonal excitation geometry, we have achieved a nearly complete elimination of laser background scattering. This in turn allows us to show resonantly controlled light emission of quantum dots in the cavity including (a) Rabi flopping using pulse control, (b) direct observation of Mollow triplets in the frequency domain, and (c) simultaneously measured first-order and second order photon-photon correlations. Keywords: Quantum Optics, Quantum Dots, Coherent control
\end{abstract}

\section{INTRODUCTION}

Semiconductor quantum dot (QD) "artificial atoms" possess optical properties similar to those of isolated atoms [1]. In addition to a much larger transition dipole moment compared to their natural counterpart because of their mesoscopic size, QDs can be tailored to give rise to desired electronic properties. These developments bring about exciting possibilities for quantum optical control in solid-state nanostructures and to harness these properties for novel optical device applications, including the current topic of quantum information science. For example, QDs could be used to realize deterministic solid-state single photon sources [2] and qubit-photon interfaces [3]. Advances in high-Q cavities have shown that not only can the spontaneous emission rate be dramatically increased by the Purcell effect [4], but emission can be reversed in the strong coupling regime $[5,6]$.

Despite these efforts, however, quantum dot-based cavity quantum electrodynamics (QED) has until now lacked an ingredient essential to the success of atomic cavity QED, namely the ability to truly resonantly manipulate the two-level system [2]. Previous approaches could at best populate the dot in one of its excited states, which subsequently relaxes in some way to the emitting ground state. This incoherent relaxation has been addressed theoretically [7], and experimentally [8] and alternatives such as the Raman-assisted lambda system have been explored [9], as it is very challenging to differentiate the resonance fluorescence from same-frequency laser scattering off defects and other contaminants. In quantum dots without cavities, coherent manipulation of ground-state excitons has nonetheless been achieved with a number of techniques including differential transmission [10], differential reflectivity [11], four-wave mixing [12], photodiode spectroscopy [13], and Stark-shift modulation absorption spectroscopy [14]. However, none of these is able to collect and use the actual photon emission, which limits their use in many potential applications of QDs.

Here we report the first measurement of resonance fluorescence from a single quantum dot. Using a waveguide cavity approach with orthogonal excitation/detection we fully discriminate the resonant emission from background laser scattering. Strongly-driven resonance fluorescence is manifested in a variety of measurements of the emitted light: the first order correlation function, obtained by interferometry, becomes oscillatory with increasing driving field and represents the time-domain analog of the fluorescent Mollow triplet [15]. Likewise, the second-order correlation function, as measured with a fast Hanbury-Brown and Twiss setup exhibits oscillations which are the direct consequence of the coherent oscillations in the dot. Finally, the Mollow triplet is also resolved spectrally [16].

Advances in Photonics of Quantum Computing, Memory, and Communication III, edited by Zameer U. Hasan,

Alan E. Craig, Philip R. Hemmer, Charles M. Santori, Proc. of SPIE Vol. 7611, 761100

(C) 2010 SPIE $\cdot$ CCC code: 0277-786X/10/\$18 · doi: 10.1117/12.847066

Proc. of SPIE Vol. 7611 761100-1 


\section{EXPERIMENTAL SETUP}
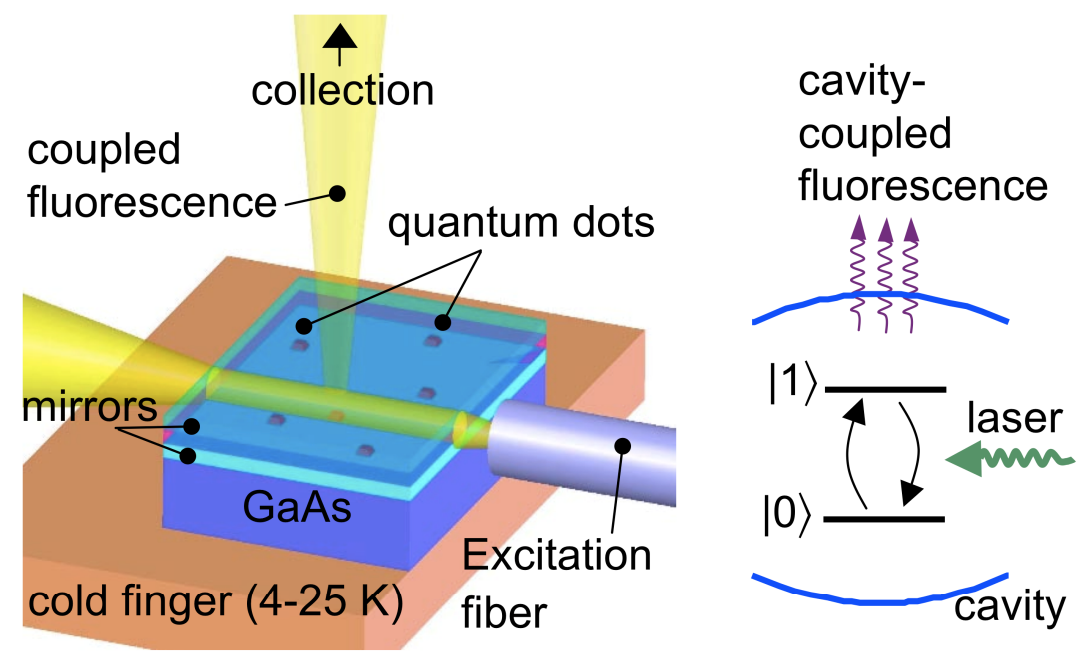

Figure 1. Schematic of the experimental setup. A single mode fiber is brought into close proximity of the cleaved edge of the semiconductor sample. The laser is guided through the chip and excites single QDs along its propagation path. Emission from the laser-excited QDs, at the same frequency as that of the laser, is coupled to a vertical cavity mode and thus separated from the laser and its background scattering.

QDs were grown by molecular beam epitaxy on GaAs, embedded in a planar microcavity consisting of two Distributed Bragg Reflectors (DBRs). The cavity was designed to have a principal resonance around $920 \mathrm{~nm}$ at low temperature with moderate quality factor. The purpose of the cavity is twofold: (i) to enhance the emission of the quantum dots along the direction of collection, and (ii) to create waveguiding for the incoming excitation beam. The unwanted scattering in the vertical direction associated with this beam is therefore minimized. Ideally, excitation and emission are decoupled due to the cavity. Fig. 1 schematically illustrates our setup. The sample is maintained at liquid He temperature while the excitation fiber, mounted on a three-axis inertial positioner in vacuum (but at room temperature) is brought into close proximity with the sample edge. The sample was carefully cleaved to minimize direct scattering at the edge and to couple the light effectively into the sample.

When the fiber is positioned at the optimal height, the incoming beam is able to efficiently excite the quantum dots in the cavity. The QD emission, at the same frequency as that of the laser, is detected in the direction perpendicular to the sample using a high-resolution microscope objective and an imaging spectrometer equipped with a nitrogen-cooled CCD camera.

\section{MEASUREMENTS}

\subsection{Spectral imaging}

Figure 2 shows spectral images that represent the emission intensity as a function of emission frequency and as a function of position (corresponding to the position along the spectrometer slit). Residual background laser scattering appears as a line because it is spatially delocalized. It is faintly visible in these (purposefully descaled) images. The emission from the single QD on the other hand is both spatially and spectrally localized and appears as a bright spot. We have measured extinction ratios as a high as 1:1000 for the background laser scattering, in comparison to the peak 
emission from a single QD. The extracted lineshape is shown below the spectral images, revealing a linewidth of less than $3 \mu \mathrm{eV}$. This lineshape represents the excitation spectrum of resonance fluorescence, which exhibits saturation and power broadening, characteristic for a resonantly excited two-level system.

\subsection{Steady-state properties of resonance fluorescence}

Saturation and power broadening are observed when measuring the total emission intensity and the linewidth, respectively, as a function of applied laser intensity. Such measurements are shown in Fig. 3 for the same QD as in Fig. 2. The data is well-described by the theoretical functions obtained from solving the optical Bloch equations,
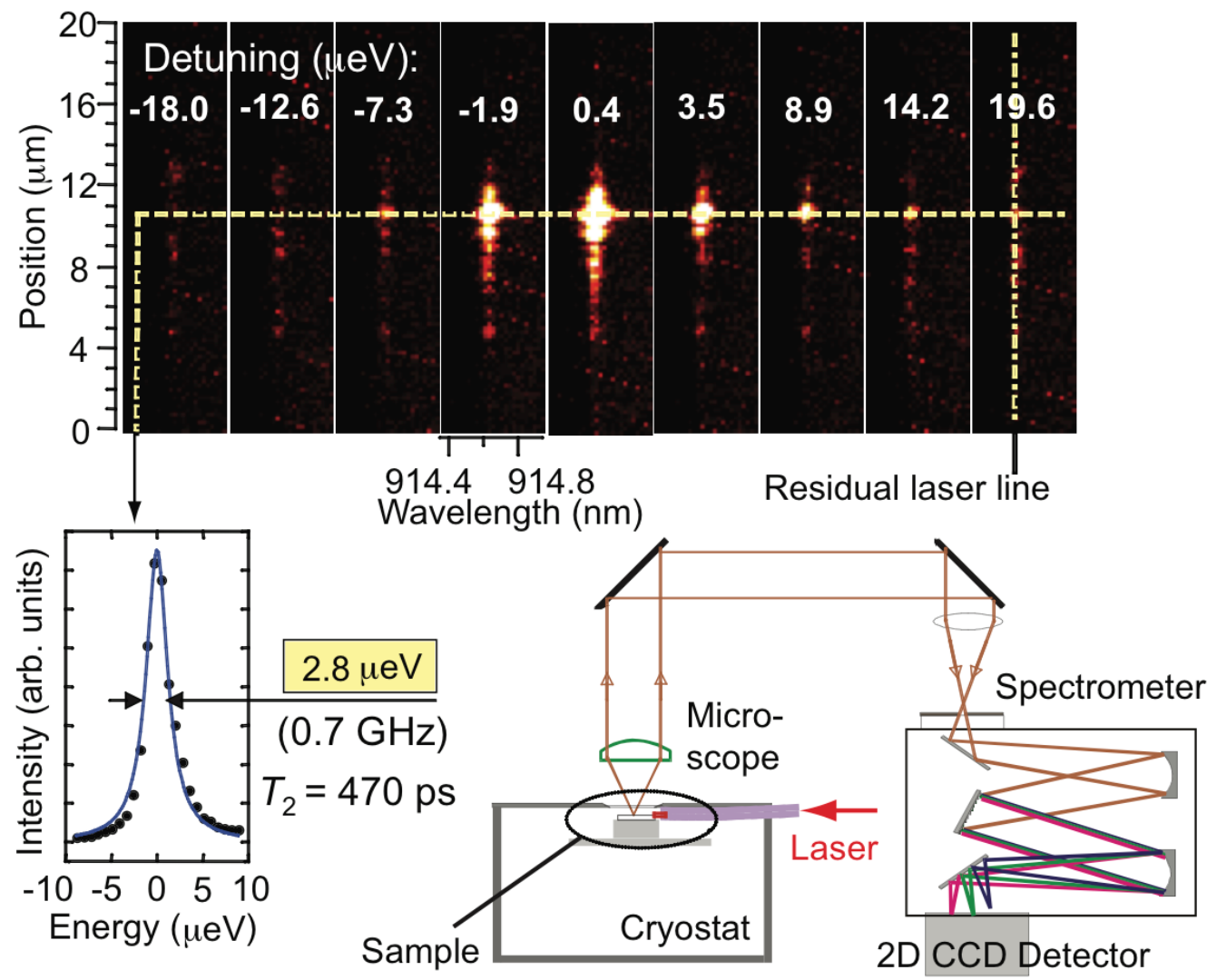

Figure 2. Resonance fluorescence from a single QD, revealed in spectral images recorded as the laser is scanned in frequency. When the laser is on resonance with the single QD, strong and spatially localized emission is observed. The background laser scattering is faintly visible as a straight line.

$$
\begin{aligned}
& \frac{d}{d t} n(t)=-i \frac{\Omega}{2}\left(\alpha(t)-\alpha^{*}(t)\right)-\frac{n(t)}{T_{1}} \\
& \frac{d}{d t} \alpha(t)=-i \frac{\Omega}{2}(n(t)-m(t))+i \alpha(t) \Delta \omega-\frac{\alpha(t)}{T_{2}}
\end{aligned}
$$

in which $n(t)=\operatorname{Tr}\{\rho(t)|1\rangle\langle 1|\}$ and $m(t)=\operatorname{Tr}\{\rho(t)|0\rangle\langle 0|\}$ are the populations of a two level system with states $|0\rangle$ and $|1\rangle$, and $\alpha(t)=\operatorname{Tr}\{\rho(t)|0\rangle\langle 1|\}$ denotes the coherence between them. $\rho(t)$ is the density operator and $T_{1}$ and $T_{2}$ are the diagonal and off-diagonal phenomenological damping constants, respectively. The Rabi frequency, $\Omega=\mu E_{0}$, quantifies the strength of the laser/two-level system interaction. $\mu$ is the transition dipole moment, and $E_{0}$ the laser electric field amplitude. In steady-state we find, 


$$
\begin{aligned}
& n_{\infty}(\Delta \omega)=\frac{1}{2} \frac{\Omega^{2} T_{1} / T_{2}}{\Delta \omega^{2}+T_{2}^{-2}+\Omega^{2} T_{1} / T_{2}} \\
& \alpha_{\infty}(\Delta \omega)=\frac{i \Omega}{2} \frac{1 / T_{2}+i \Delta \omega}{\Delta \omega^{2}+T_{2}^{-2}+\Omega^{2} T_{1} / T_{2}}
\end{aligned}
$$

Equation (3) was used to plot the solid lines in Fig. 3. As saturation occurs, the strong excitation regime is entered and the two-level system is no longer most of the time in its ground state. Instead, the strong laser causes both the population inversion as well as the coherence to oscillate in time. To observe these oscillations we need to measure the emission spectrum rather than the excitation spectrum. However, the resolution of the spectrometer used in the measurements of Fig. 2 is not sufficient for this purpose and we resort to Fourier spectroscopy or scanning Fabry-Perot interferometry.
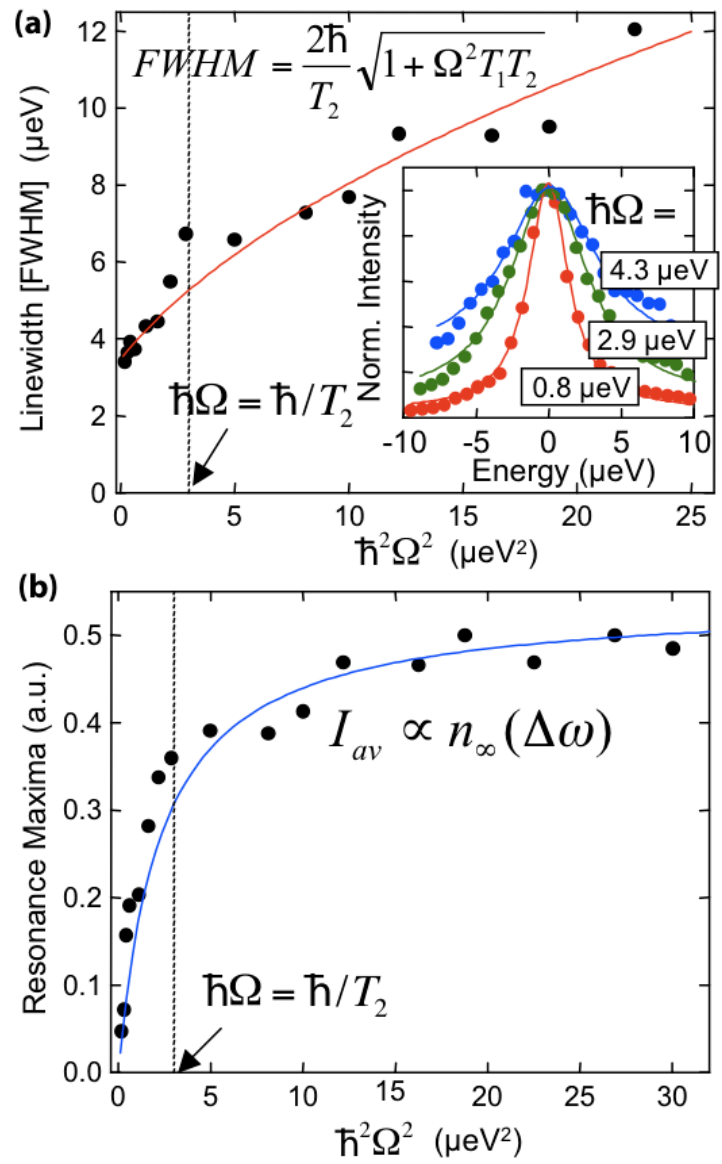

Figure 3. (a) Linewidth of the resonance fluorescence excitation spectrum as a function of square Rabi energy, proportional to the applied laser intensity. (b) QD emission intensity when on resonance with applied laser, as a function of square Rabi energy.

\subsection{Time domain properties}

In Fourier spectroscopy, the emission is split and then interfered at a beam splitter, and the observed fringe contrast recorded as a function of time delay between the two split beams. This fringe contrast is proportional to the first order correlation function, $g(t, \tau)$, of the photons. The first order correlation function of light emitted by a two level quantum system was first computed by Mollow [17] from $g(t, \tau)=\left\langle b^{+}(t) b(t+\tau)\right\rangle$, where $b$ and $b^{+}$are the field operators which are proportional to the atomic dipole operators, $|0\rangle\langle 1|$ and $|1\rangle\langle 0|$, respectively. The result reads: 


$$
g(\tau)=\left|\alpha_{\infty}(0)\right|^{2}+\frac{n_{\infty}(0)}{2} e^{-\tau / T_{2}}+n_{\infty}(0) e^{-\tau\left(1 / T_{1}+1 / T_{2}\right) / 2}\left\{N \cos \left(\Omega^{\prime} \tau\right)+M \sin \left(\Omega^{\prime} \tau\right)\right\}
$$

where $N$ and $M$ denote constants that depend on $T_{1}, T_{2}$, and $\Omega$, and $\Omega^{\prime}=\sqrt{\Omega^{2}-\left(1 / T_{1}-1 / T_{2}\right)^{2} / 4}$. When $\Omega<<1 / T_{2}$, then $g(\tau)$ reduces to a simple exponential decay, with decay constant $T_{2}$, corresponding to a Lorentzian spectral line profile of FWHM $2 / T_{2}$. On the other hand, when $\Omega>>1 / T_{2}$, the system is in the strong excitation regime and $g(\tau)$ is oscillatory. Note that $T_{1}$ and $T_{2}$ are related through $T_{2}^{-1}=\left(2 T_{1}\right)^{-1}+\gamma$, where $\gamma$ denotes pure dephasing (i.e. loss of coherence without population decay).

Figure 4 shows the fringe contrast obtained from the emission from the same QD as in Fig. 2. Its evolution with excitation laser intensity, i.e. Rabi frequency, is as expected from theory: when the intensity is weak, i.e. $\Omega<<1 / T_{2}$, a single exponential decay is obtained. But with increasing intensity, the fringe contrast develops an oscillatory feature at frequency $\Omega^{\prime}$, defined above, which approximately equals $\Omega$ if $\Omega>>1 / T_{2}$. The oscillations can be understood as an amplitude modulation imposed on the field by the two-level system undergoing fast Rabi cycles. The system is sufficiently coherent to observe several oscillations, analogous to the observation of a distinctive Mollow triplet.

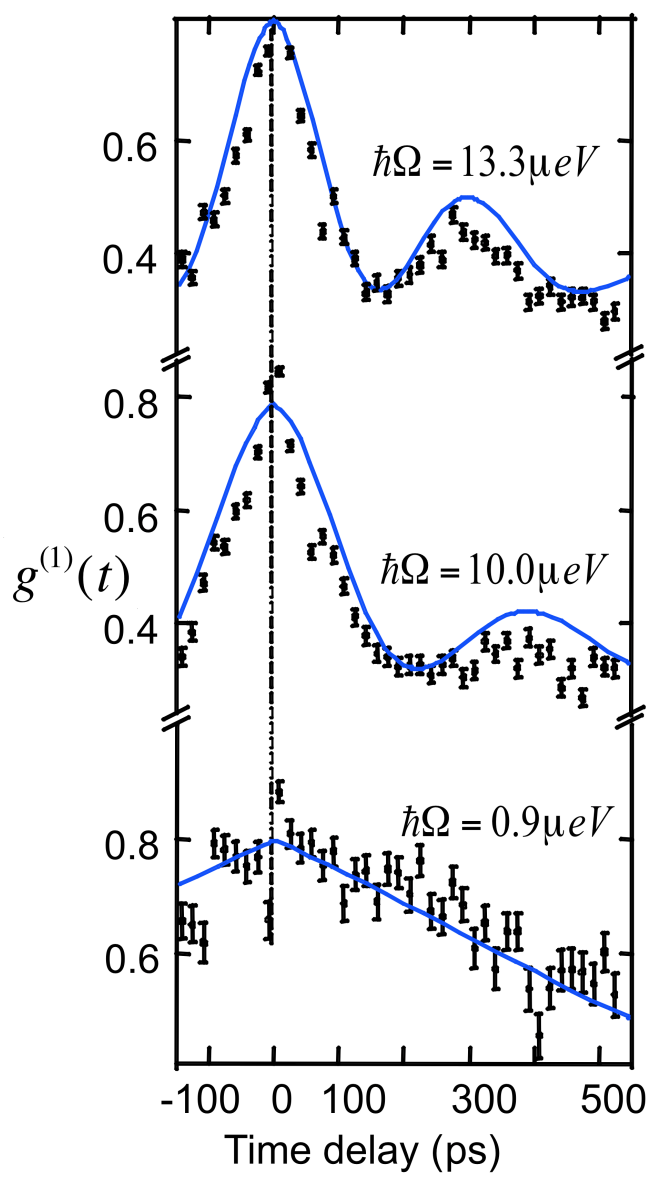

Figure 4. (a) First-order correlation measurement of the emission of the same QD as in Fig. 2 and Fig. 3. It was measured with a Mach-Zehnder interferometer. Data is shown for three values of the applied laser intensity, increasing from bottom to top. 


\subsection{Spectral properties}

We can also observe this Mollow triplet in a direct spectral measurement with a scanning Fabry Perot interferometer (FPI). Such a measurement is shown in Fig. 5 for a different QD, for various excitation intensities of the laser. The theoretical form of the triplet is obtained from the Fourier transform of $g(t, \tau)$ in Eq. (5),

$$
S(\Delta v)=\frac{n_{\infty}}{\pi}\left\{\frac{1}{2} \frac{1 / T_{2}}{(\Delta v)^{2}+1 / T_{2}^{2}}+\frac{n_{\infty}}{\Omega^{2}}\left(\frac{A \eta / 2-B(\Delta v-\mu) / 8 \mu}{(\Delta v-\mu)^{2}+\eta^{2}}+\frac{A \eta / 2-B(\Delta v+\mu) / 8 \mu}{(\Delta v+\mu)^{2}+\eta^{2}}\right)\right\}
$$

where $n_{\infty}$ is the steady-state population, $\Delta v$ is the detuning of the FPI from the resonant frequency, and the constants, written out explicitly are

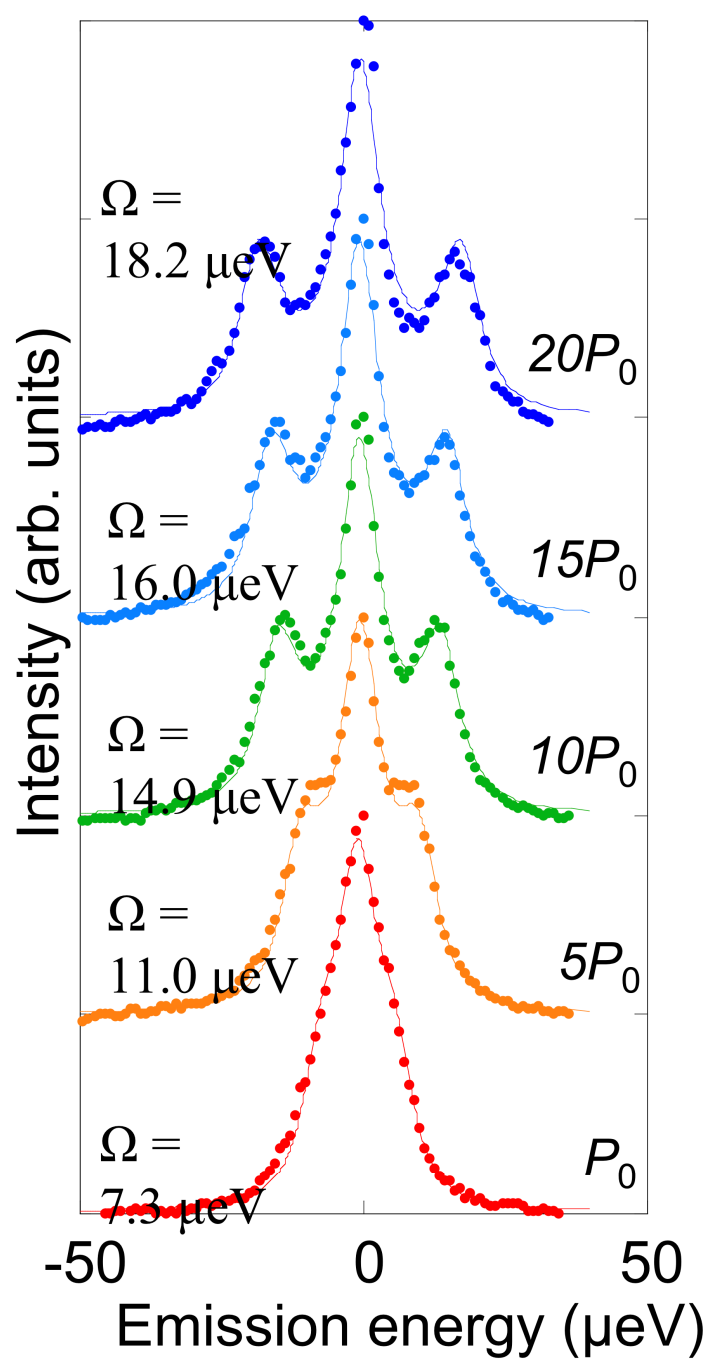

Figure 5. Resonance fluorescence spectrum measured with a scanning FPI. The applied laser intensity is increasing from bottom to top.

$$
\begin{gathered}
A=\Omega^{2}+\left(1 / T_{1}-1 / T_{2}\right) / T_{1} \\
B=2 \Omega^{2}\left(3 / T_{1}-1 / T_{2}\right)-2\left(1 / T_{1}-1 / T_{2}\right)^{2} / T_{1}
\end{gathered}
$$




$$
\begin{gathered}
\eta=\left(1 / T_{1}+1 / T_{2}\right) / 2 \\
\mu=\sqrt{\Omega^{2}+\left(1 / T_{1}-1 / T_{2}\right)^{2}}
\end{gathered}
$$

This power spectrum has a central peak with two side bands that are separated from the central peak by the Rabi frequency when significantly in the strong excitation regime. This triplet can also be viewed in the dressed states picture as the sum of four total transitions between pairs of dressed states from neighboring rungs of a periodic latter. Because the dressed states latter is periodic in the laser frequency (not the natural frequency of the transition), two of the four transitions are at the laser frequency and make up the central peak that is thus about twice as strong as the sidebands.

\subsection{Photon statistics}

Finally, another distinctive feature of resonance fluorescence are found in its photon statistics. Because the emission originates from a driven two-level quantum system, the photon statistics are significantly modified in comparison to a non-resonantly excited emitter. In particular, the oscillations undergone by the two-level system are impregnated onto the time-delay between emitted photons.

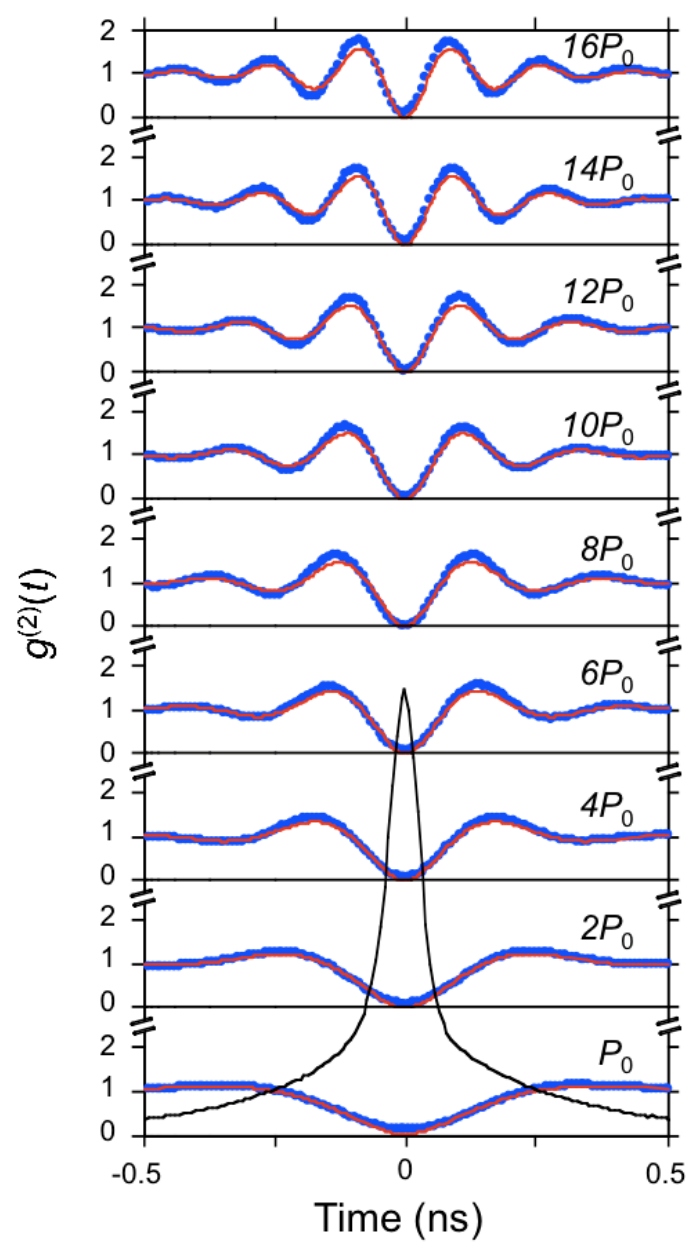

Figure 6. Second-order correlation measurement of the resonance fluorescence. It was measured with a HanburyBrown and Twiss setup using two fast avalanche photodiodes. Data is shown for increasing values of the applied laser intensity from bottom to top.

Theoretically, the photon statistics are described by the second order correlation function 


$$
g^{(2)}(\tau)=1-\operatorname{Exp}[-\eta|\tau|\}\left\{\cos (\mu|t|)+\frac{\eta}{\mu} \sin (\mu|t|)\right\}
$$

It represents the probability of obtaining a second photon at time $t=\tau$ when a first photon was obtained at time $t=0$. Experimentally, this probability is measured by a Hanbury-Brown and Twiss setup that uses a beam splitter and two detectors. To be able to resolve the fast oscillations occurring due to Rabi frequencies of several $\mathrm{GHz}$, we use ultrafast avalanche photodetectors that have a time resolution on the order of $50 \mathrm{ps}$.

Measurements of the photon statistics are shown in Fig. 6 for various intensities of the applied laser. When this intensity is low an anti-bunching dip is observed, characteristic for a single quantum emitter. As the intensity is increased, and oscillatory behavior develops, with a frequency corresponding to the Rabi frequency. These measured oscillations are in fact identically the time-domain Rabi oscillations described by Torrey's solutions of the Bloch equations. This is because a first detection event can be viewed as a projection of the two-level system into its ground state, from which it then evolves according to Eqs (1) and (2). The second photon, emitted randomly within a time approximately equal to the lifetime of the upper state thus "maps out" the oscillatory function of Eq. (7) and leads to the data of Fig. 6. These measurements have been corrected for background emission as well as a slow component in the time response function of the avalanche photodetectors. This response function, measured with a ps-pulsed laser, is the bell-shaped curve in Fig. 6. Even though the laser background scattering is negligible in the low excitation regime, its contribution increases as the strong excitation regime is entered because the emission from the two-level system saturates but the laser intensity continues to increase.

\subsection{Pulsed Rabi flopping}

Rabi oscillations have also been measured directly in the time-domain using excitation with a ps pulsed laser. The result of these measurements are shown in Fig. 7; a full Rabi cycle is obtained. Pulsed Rabi oscillations in the ground state have also been obtained in emission by time-gating from InAs QDs [18] and by a V-groove waveguide excitation in GaAs quantum wires [19].

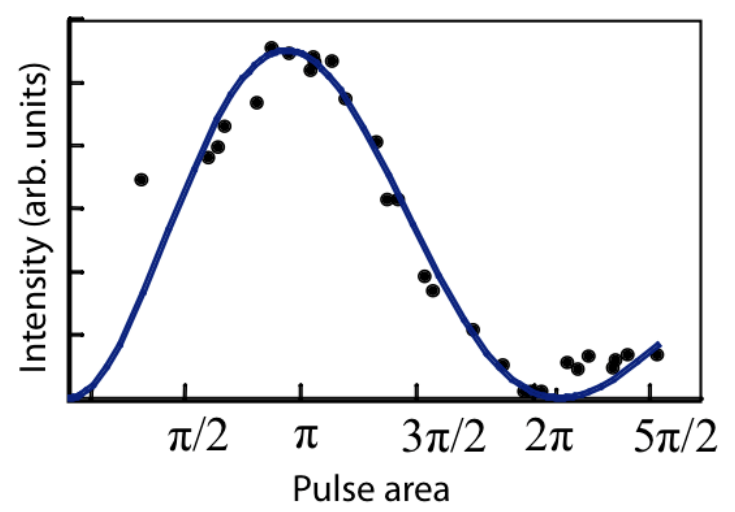

Figure 7. Rabi oscillations under pulsed resonant excitation of a single QD. The total emission is plotted as a function of the pulse area, proportional to the square root of the time-integrated Rabi frequency.

Using a three dimensional microcavity instead of a simple planar structure would further enable advanced experiments relying on cavity QED effects, many of which have been proposed for quantum information processing applications and realized in atomic systems. This might be achieved straightforwardly with all-epitaxial microcavities, that possess a bulk morphology ideally suited to introduce a waveguided laser [20].

\subsection{Conclusions}

In conclusion, our measurements, in which QDs are laterally excited in a microcavity, realize the goal of resonant coherent control of the excitonic ground state while simultaneously collecting its fluorescence. Using this method in concert with interferometry and second-order correlation measurements, we achieve the first observation, in a driven solid-state two-level system, of resonance fluorescence in the strong excitation regime. Background-free resonant measurements offer new coherent control capabilities resembling those available for manipulating trapped atoms and 
ions, yet in a monolithic, scalable solid-state system. Other promising approaches to coherent optical manipulation of the ground state of InAs QDs include differential transmission [21] and Stark shift modulation spectroscopy [22]. Resonance fluorescence side-bands have also been measured by spectral filtering with a Fabry-Perot interferometer from InAs QDs [23] as well as from single molecules [24]. Resonant excitation methods are promising for single photon indistiguishability [25] and coherent optical manipulation of quantum states [26,27].

\section{REFERENCES}

[1] M. Bayer et al., "Hidden symmetries in the energy levels of excitonic 'artificial atoms'", Nature 405, 923-926 (2000).

[2] J. McKeever et al., "Deterministic Generation of Single Photons from One Atom Trapped in a Cavity", Science 303, 1992 (2004).

[3] W. Yao, R.B. Liu, and L.J. Sham, "Theory of Control of the Spin-Photon Interface for Quantum Networks", Phys. Rev. Lett. 95, 030504 (2005).

[4] J. M. Gerard et al., "Enhanced Spontaneous Emission by Quantum Boxes in a Monolithic Optical Microcavity", Phys. Rev. Lett. 81, 1110 (1998).

[5] T. Yoshie et al., "Vacuum Rabi splitting with a single quantum dot in a photonic crystal nanocavity", Nature (London) 432, 200 (2004).

[6] J. P. Reithmaier et al., "Strong coupling in a single quantum dot-semiconductor microcavity system", Nature (London) 432, 197 (2004).

[7] A. Kiraz, M. Atature, and A. Imamoglu, "Quantum-dot single-photon sources: Prospects for applications in linear optics quantum-information processing", Phys. Rev. A 69, 032305 (2004).

[8] C. Santori et al., "Indistinguishable photons from a single-photon device", Nature (London) 419, 594 (2002).

[9] D. Fattal, R. Beausoleil, and Y. Yamamoto, "Coherent single-photon generation and trapping with imperfect cavity QED systems", arXiv:quant-ph/0606204.

[10] T. H. Stievater et al., "Rabi Oscillations of Excitons in Single Quantum Dots" Phys. Rev. Lett. 87, 133603 (2001).

[11]T. Unold et al., "Optical Stark Effect in a Quantum Dot: Ultrafast Control of Single Exciton Polarizations", Phys. Rev. Lett. 92, 157401 (2004).

[12] P. Borri et al., "Ultralong Dephasing Time in InGaAs Quantum Dots", Phys. Rev. Lett. 87, 157401 (2001).

[13] A. Zrenner et al., "Coherent properties of a two-level system based on a quantum-dot photodiode", Nature (London) $418,612(2002)$.

[14]B. Alen et al., "Stark-shift modulation absorption spectroscopy of single quantum dots", Appl. Phys. Lett. 83, 2235 (2003).

[15] A. Muller et al., "Resonance Fluorescence from a Coherently Driven Semiconductor Quantum Dot in a Cavity", Phys. Rev. Lett. 99, 187402 (2007).

[16]E. B. Flagg et al., "Resonantly driven coherent oscillations in a solid-state quantum emitter", Nature Phys. 5, 203207 (2009).

[17] B. R. Mollow, “Power Spectrum of Light Scattered by Two-Level Systems”, Phys. Rev. 188, 1969 (1969).

[18] K. Kuroda et al., "Final-state readout of exciton qubits by observing resonantly excited photoluminescence in quantum dots", Appl. Phys. Lett. 90, 051909 (2007).

[19] R. Melet et al., "Resonant excitonic emission of a single quantum dot in the Rabi regime", Phys. Rev. B 78, 073301 (2008)

[20] A. Muller et al., "Self-Aligned All-Epitaxial Microcavity for Cavity QED with Quantum Dots", Nano Lett. 6, 2920 (2006).

[21] X. Xu et al., "Coherent Optical Spectroscopy of a Strongly Driven Quantum Dot", Science 317, 929 (2007).

[22] G. Jundt et al., "Observation of Dressed Excitonic States in a Single Quantum Dot", Phys. Rev. Lett. 100, 177401 (2008).

[23] A.N. Vamivakas, Y. Zhao, C.-Y. Lu, and M. Atatüre, "Spin-resolved quantum-dot resonance fluorescence", Nature Phys. 5, 198-202 (2009).

[24]G. Wrigge et al., "Efficient coupling of photons to a single molecule and the observation of its resonance fluorescence", Nature Phys. 4, 60 (2008).

[25] S. Ates etl al., "Post-Selected Indistinguishable Photons from the Resonance Fluorescence of a Single Quantum Dot in a Microcavity", Phys. Rev. Lett. 103, 167402 (2009). 
[26] A. Muller et al., "Emission Spectrum of a Dressed Exciton-Biexciton Complex in a Semiconductor Quantum Dot", Phys. Rev. Lett. 101, 027401 (2008).

[27] A. Muller et al., "Creating Polarization-Entangled Photon Pairs from a Semiconductor Quantum Dot Using the Optical Stark Effect”, Phys. Rev. Lett. 103, 217402 (2009). 